\title{
Digital implementation of a self-triggered control approach for a mechatronic platform: experimental results
}

\section{Carlos Santos, Javier Echevarría, Felipe Espinosa*, Marta Marrón, Cristina Losada and Daniel Pizarro}

\author{
Electronics Department, \\ Engineering School, \\ University of Alcalá, \\ Campus Universitario s/n 28805, \\ Alcalá de Henares, Madrid, Spain \\ Email: carlos.santos@uah.es \\ Email: j.echevarria@edu.uah.es \\ Email: felipe.espinosa@uah.es \\ Email: marta.marron@uah.es \\ Email: cristina.losada@uah.es \\ Email: daniel.pizarro@uah.es \\ *Corresponding author
}

\begin{abstract}
This paper addresses the design and presents the experimental results of an aperiodic remote-controlled mechatronic plant using a MiniDK2 electronic development board. We compare the classic periodic control solution with our self-triggered approach. The triggering mechanism consists of evaluating if the measurement error exceeds a predefined value. This measurement error is defined as the difference between the output signal of a model with and without a sample-and-hold that is activated only at the triggering instants. The minimum inter-execution time is the reference period and the maximum time is set by the designer, guaranteeing the stability of the closed loop system. At each new triggering instant, the remote controller receives a new measurement of the system and sends the actuation signal to the mechatronic plant.
\end{abstract}

Keywords: aperiodic digital control; self-triggered control; remote control; MiniDK2 development board.

Reference to this paper should be made as follows: Santos, C., Echevarría, J., Espinosa, F., Marrón, M., Losada, C. and Pizarro, D. (2018) 'Digital implementation of a self-triggered control approach for a mechatronic platform: experimental results', Int. J. Intelligent Machines and Robotics, Vol. 1, No. 1, pp.60-78.

Biographical notes: Carlos Santos received his BS degree in Telecommunications Engineering in 2010, MSc in Electrical Engineering in 2011 and PhD degree in 2016, all from the University of Alcala (UAH), Spain. $\mathrm{He}$ is a member of the Electronics Engineering Applied to Intelligent Spaces and Transport Group (GEINTRA). His research interest focuses on the field of fusion algorithms, trajectory generation for navigation in mobile robotics and varying-time sampling control techniques. 
Javier Echevarría received his MS degree in Electronics by the University of Alcala (UAH), Spain. He has participated in electronic control projects with the Electronics Department of the UAH. His current professional experience is related with control systems.

Felipe Espinosa received his MS degree from the Polytechnic University of Madrid (UPM), Spain, and PhD degree from the University of Alcala (UAH), both in Telecommunication, in 1991 and 1999 respectively. He became a Full Professor in 2016 in the Electronics Department with the UAH, regularly involved in electronic control and automation subjects in several post-degree programmes. His current research interests include network control systems, event-based control, wireless network systems and sensorial integration applied to intelligent transportation systems and intelligent environments.

Marta Marrón received her PhD in Intelligent Systems Advanced Electronics from the University of Alcalá (UAH), Spain, in 2008. She was an Assistant Professor from 2001 to 2008 and Associate Professor since 2009 within the Department of Electronics in the UAH. She is a member of the Electronics Engineering Applied to Intelligent Spaces and Transport Group (GEINTRA). Her research interests include multisensor indoor localization (intelligent spaces), scene understanding, human behaviour analysis, computer vision, probabilistic algorithms, embedded and electronics control systems and robotics in general, and personal mobile robots in particular applied to assistive technologies.

Cristina Losada received her BS degree in Telecommunications Engineering in 2004, and her PhD in Intelligent Systems Advanced Electronic in 2010, both from the University of Alcalá, Spain. From 2011 to 2016, she was a PhD Assistant Lecturer in the Electronics Department with the UAH. Since 2016, she has been an Associate Professor in the same department. Her research interests are focused on computer vision, human behaviour analysis, intelligent spaces and intelligent sensor systems. She has authored 12 publication in international journals, and more than 30 publications in conference proceedings.

Daniel Pizarro received his $\mathrm{PhD}$ degree in Electrical Engineering in 2008 from the University of Alcala (UAH). In 2005-2012, he was an Assistant Professor and member of the GEINTRA group at the UAH. From 2013 to 2015, he was an Associate Professor at the Université d'Auvergne and member of ALCoV research group. Since 2015, he has been an Associate Professor at the UAH. His research interests are in optimisation and computer vision, including image registration and deformable reconstruction, and their application to minimally invasive surgery.

\section{Introduction}

Since the first published studies on event-based control (EBC), as an alternative to time-based control (Gupta, 1963; Aström, 1999; Arzén, 1999), there is an extensive bibliography related to this topic in the scientific literature, from which it can be highlighted journal papers as Heemels (2008), Lehmann and Lunze (2009) or Heemels (2012), books as Miskowicz (2015) or (Lunze, 2014) and even a specific conference as 
the 'Conference on Event Based Control Communication and Signal Processing', whose first edition took place in 2015.

Against the classic digital control, executed with a constant period $T$, the EBC executes the control law only when it is required, relaxing sensors, actuators and the global execution time of the control device.

EBC is particularly interesting in network control applications (Hespanha et al., 2007; Gupta, 2010), where the sensors, the digital controller, or both are wireless connected to the controlled process (Figure 1). This way, a more efficient use of the shared resources is achieved by the nodes of the network, such as the communication channel.

Figure 1 Network control system divided into three nodes (see online version for colours)

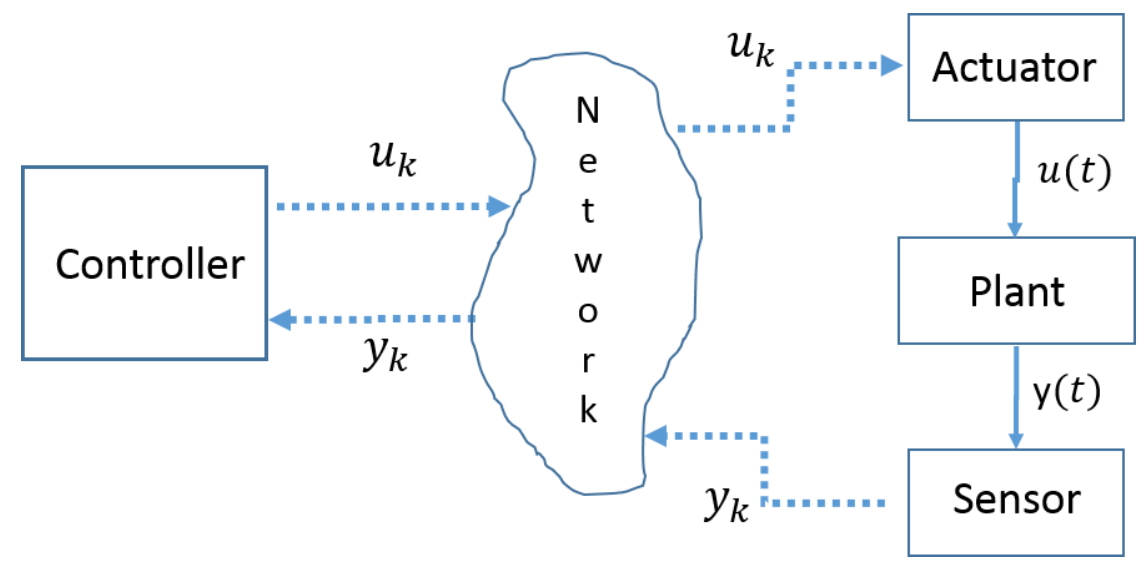

Note: Actuator (input $u$ ), sensor (output $y$ ) and controller.

There are two main approaches in EBC: event-triggered (ETC) and self-triggered (STC). The first one has a reactive behaviour, so that when a sensor detects a significant change in a measured variable with respect to a prespecified value, it establishes a new communication (triggering instant) with the controller (Mazo and Tabuada, 2008; Heng et al., 2015). The second approach has a proactive behaviour, so that the triggering instant is predicted depending on the last actuation on the plant and its dynamics (Mazo et al., 2009; Anta and Tabuada, 2010).

The interest between each alternative relies on the application scenario. A clear example of ETC is monitoring robots using networked sensors, where the triggering mechanism is on-boarded (Martinez-Rey et al., 2016a) or in the remote sensor (Martinez-Rey et al., 2016b). The remote EBC of a robot that incorporates its own sensory system (Santos et al., 2014) is a clear example of STC.

There are two important aspects to take into account in the STC design process: on the one hand the triggering mechanism (condition of the next event) and on the other hand the stability study. Usually this questions are achieved using Lyapunov functions evaluated in continuous time. These functions intrinsically guarantee the stability (practical or asymptotic) of the system and their temporal evolution allow establishing the triggering mechanism (Santos et al., 2015).

The increasing interest in EBC solutions leads to promote the introduction of these techniques in the syllabus of control subjects in engineering studies. In the bachelor's degree, studies traditionally have prevailed the digital control techniques with constant 
sampling (discrete-time control) applied to processes that respond to a linear model, and where the stability study is approached with techniques such as the root locus and the frequency domain response (Ogata, 1995; Fadali and Visioli, 2012; Losada et al., 2016). Thus, nonlinear control and stability study based on Lyapunov fucnctions (Slotine and Li, 1991; Dutton, 1997; Sastry, 1999; Khalil, 2013) are discussed at higher levels of education.

This paper presents an approximation to the STC of a mechatronics plant comparing it with its periodic control version. In addition, we present a stability study that does not require Lyapunov functions. The global objective is to analyse the pros and cons of both techniques using as the plant under study a mechatronics platform, remote-controlled by an evaluation electronic board based on a general-purpose microcontroller.

The mechatronic plant is described in Losada et al. (2016). It basically consists of a motion actuator (specifically a DC-motor) with its electric drive and sensory interface. The electronic device that implements the STC proposal is an evaluation board based on a Cortex-M3. A local area network connects these elements, as is shown in the diagram of Figure 2. From the control point of view, we model the communication channel as a part of the plant to be controlled.

Figure 2 Schematic of the STC remote control of the mechatronic platform under study (see online version for colours)

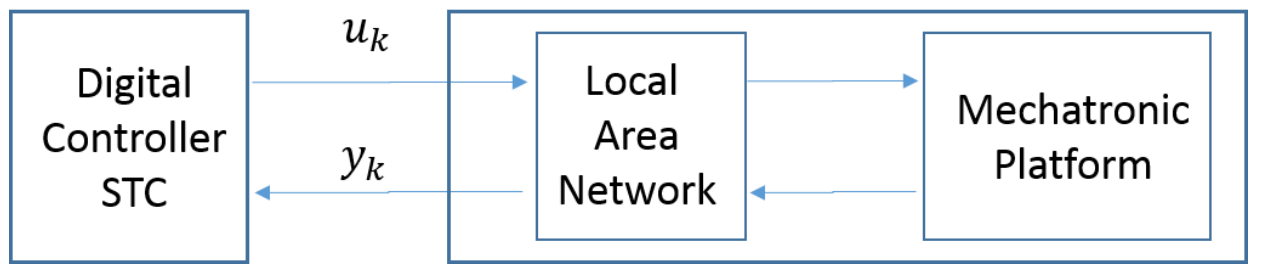

This case study is commonly used in academic contexts, especially in laboratories with a small number of systems (plants to control) connected to a LAN or a WLAN and numerous workstations in which students test their proposal in the board connected to the same network.

\section{Notation}

Positive real values $\mathbb{R}^{+}$; natural values $\mathbb{N}$; positive natural values $\mathbb{N}^{+}$; reference sampling period $\mathrm{T}$, periodic evaluation time $t_{T}=n \cdot T, n \in \mathbb{N}$; aperiodic evaluation time or triggering time $t_{k}$, in general $t_{k+1}-t_{k} \geq T$; representation of periodically evaluated signals $x_{T}=x\left(t_{T}\right)$ or aperiodically $x_{k}=x\left(t_{k}\right)$.

\section{Self-triggered control design}

We use the periodic control procedure applied to a mechatronic plant as starting point, where the controller and the plant are nodes of the same LAN. In Figure 3 we represent the feedback system where the channel delay is included in the plant model. 
Figure 3 Model of the periodic remote control of the mechatronic plant (see online version for colours)

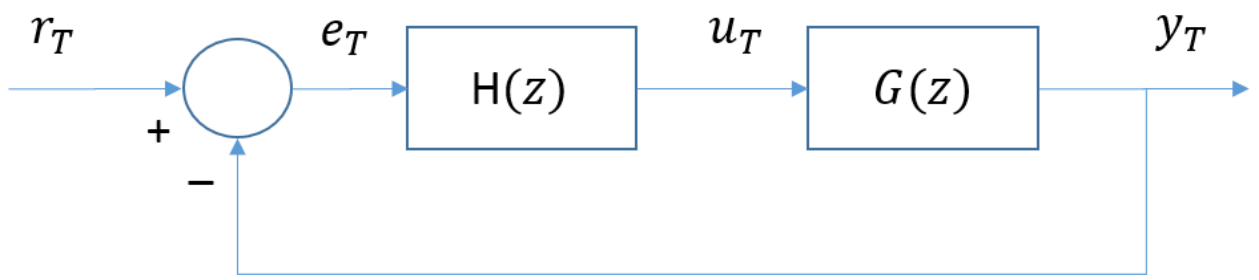

We fix the sample period in the plant identification and modelling stage, depending on the plant dynamics and the channel delay. From the plant linear model $G(z)$, the design specifications are set, and the controller model $H(z)$ is obtained. The relevant variables in the controlling process are: reference $r_{T}$, tracking error $e_{T}$, actuator signal $u_{T}$, and output signal $y_{T}$.

The diagram in Figure 4 represents the implemented aperiodic control strategy. In this solution, the real plant receives a control law update only at the triggering times $t_{k}$, we model this behaviour using a sample-and-hold $(\mathrm{S} / \mathrm{H})$ implementation activated at triggering instants. The signal $y_{T}$ is the response of the discretised plant $G(z)$ to the periodic input $u_{T}$; while $\bar{y}_{T}$ is the response of $G(z)$, evaluated each $\mathrm{T}$, to the aperiodic input $u_{k}$. This actuation signal is updated at the triggering instants $t_{k}$, and it is sent to the mechatronic plant connected to the LAN. The real plant responds with the measurement $y_{k}$. The selector block chooses the feedback variable $y$ to be compared with the reference $r_{T}$ in order to obtain the tracking error $e_{T}$. The choice depends on the value of the flag $\sigma_{T}$, i.e.,

$$
\begin{aligned}
& y=y_{T} \text { if } \sigma_{T}=0, \\
& y=y_{k} \text { if } \sigma_{T}=1 .
\end{aligned}
$$

Figure 4 Structure of the proposed aperiodic (STC) control solution (see online version for colours)

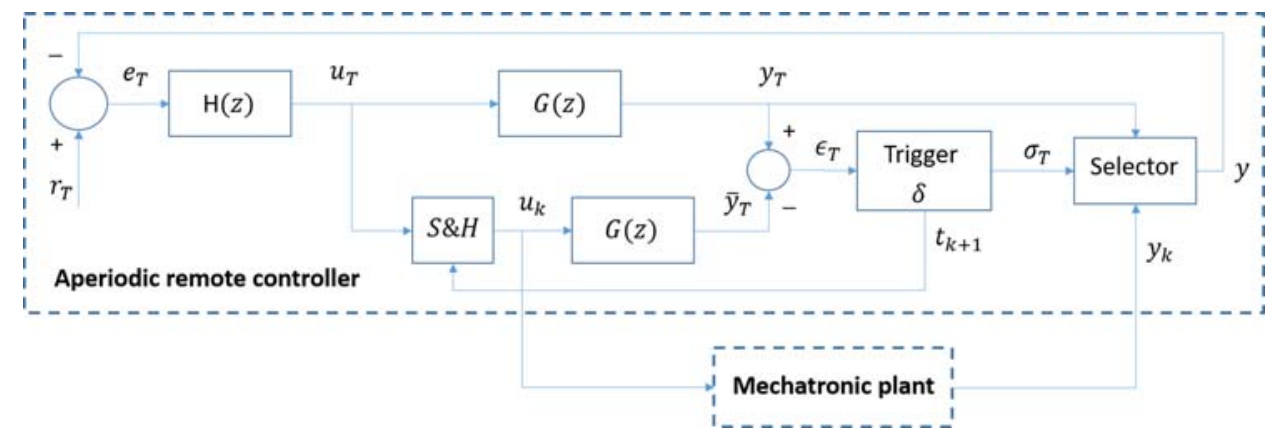

The triggering mechanism is now described. A new triggering, and therefore a new channel access, is generated when the measurement error $\in_{T}$, defined as the difference between $y_{T}$ (response of $G(z)$ to the periodic input $u_{T}$ ) and $\bar{y}_{T}$ (response of $G(z)$ to the aperiodic input $u_{k}$ ), exceeds a predefined threshold $\delta$ set by the designer. The calculation of the triggering instants is defined by: 


$$
t_{k+1}=t_{T}>t_{k}|| y\left(t_{T}\right)-\bar{y}\left(t_{T}\right) \mid>\delta ; k \in \mathbb{N} .
$$

The minimum time between triggering instants matches with the reference period: $t_{k+1}-t_{k}>T, k \in \mathbb{N}$.

When the triggering condition is violated, the flag $\sigma_{T}$ is activated during a sampling period, and the bidirectional communication between the remote controller and the mechatronic plant is established.

Our proposal is characterised by the periodical controller updating of internal variables: $e_{T}, y_{T}, \bar{y}_{T}, \in_{T}, \sigma_{T}$. Oppositely, the standard STC anticipates the next triggering, in a reference period, once the real plant output $y_{k}$ is obtained. Our solution does not liberate the digital processor to do other possible tasks but avoids a constant input applied to the PI controller for the inter-execution time.

\subsection{Stability study}

The identification process of the mechatronic plant (Losada et al., 2016), sending actions $u_{T}$ from the remote controller and receiving the plant output $y_{T}$, allows us to adapt the reference period $\mathrm{T}$ to the plant dynamics and the channel delay. Given that the plant is a first order system and the channel delay is a sampling period, the discrete model of the plant seen from the remote controller fits the expression equation (3):

$$
G(z)=\frac{\alpha}{z(z-\beta)} ; \alpha \in \mathbb{R}^{+}, 0<\beta<1 .
$$

The PI controller is designed from time response specifications of the feedback system in Figure 3 , obtaining the controller transfer function in equation (4), with zero, pole $p=1$ and gain $K$ :

$$
H(z)=K \frac{z-c}{z-1} ; K>0,0<c<1
$$

Between triggering instants the real plant stays in open loop, which makes it sensitive to disturbances. Therefore, in the design of the mentioned aperiodic control, a maximum time $t_{\max }$ between triggering instants is fixed, being this time value a multiple of the reference $T$, as shown in equation (5):

$$
t_{\max }=p \cdot T, p \in \mathbb{N}^{+} .
$$

Therefore, the value of $t_{k+1}$ in expression equation (2) is bounded by $t_{\max }$ equation (6):

$$
t_{k+1}=t_{\max }>t_{T}>t_{k}|| y\left(t_{T}\right)-\bar{y}\left(t_{T}\right) \mid>\delta ; k \in \mathbb{N} .
$$

In general, STC systems stability is studied in the continuous time domain using the Lyapunov method (Heemels et al., 2008, 2012; Mazo and Tabuada, 2008; Mazo et al., 2009; Santos et al., 15). In this paper, we assume that the discrete control system in Figure 3 is stable, and the effect on the plant of increasing the time between communications is equivalent to discretise it with a sampling period $T_{x} \geq T$. Thus, for the worst case $T_{x}=T_{\max }$, the discretised plant model is $G_{x}(z)$ If the system in Figure 3 is stable for $T_{x}=T$ and for $T_{x}=T_{\max }$, it is also stable for the intermediate values $T<T_{x}<$ 
$T_{\max }$. This facilitates the stability analysis of the system under study applying known techniques applicable to linear systems (Jury test, root locus, etc.).

Thus, in case of complying:

$$
T x>5 \tau+L,
$$

where $\tau$ is the time constant of the plant and $L$ is the delay of the channel, the model $G_{x}(z)$ is reduced to:

$$
G_{x}(z)=\frac{\theta}{z}, \theta=\frac{\alpha}{1-\beta}
$$

In order to evaluate the stability of the feedback system, without modifying the original controller $H(z)$ and with the plant model $G_{x}(z)$, it is enough to analyse the roots of the characteristic equation (9):

$$
1+K \frac{z-c}{z-1} \frac{\theta}{z}=0
$$

The roots from equation (9) are shown in equation (10):

$$
z_{1}=\frac{1-K \theta+\sqrt{(K \theta-1)^{2}+4 K c \theta}}{2}, z_{2}=\frac{1-K \theta-\sqrt{\theta+4 K c \theta}}{2}
$$

Since $\left|z_{1}\right|<\left|z_{2}\right|$, the stability of the feedback system is guaranteed if $\left|z_{2}\right|<1$.

\section{Simulation results}

Prior to the STC experimentation, the feedback system stability is tested and validated by simulation.

\subsection{Model of the mechatronic plant}

We tested the plant with the electronic system described in Section 2, we applied different signals from the remote controller in open loop, and considering the channel delay as a part of the plant model, after the identification task with $T=5 \mathrm{~ms}$, we obtained the transfer function in (11):

$$
G(z)=\frac{0.361}{z(z-0.802)}
$$

Thus, the plant model is characterised by the following parameters: time constant of $22.6 \mathrm{~ms}$, static gain of $1822 \mathrm{rpm} /$ input-code and channel delay of $5 \mathrm{~ms}$.

\subsection{Stability study of the aperiodic solution}

Establishing as specifications for the periodically evaluated controller design (reference control), an overdamped response with a dominant time constant of $22.6 \mathrm{~ms}$, the controller transfer function $H(z)$ results as shown in equation (12): 


$$
H(z)=0.361 \frac{z-0.802}{z-1}
$$

Nevertheless, maintaining the control algorithm $H(z)$ in equation (12), an aperiodic access to the real plant input and output $\left(u_{k}, y_{k}\right)$ changes its discretised behaviour with respect to the identified in equation (11), as well as the temporary response of the closed control loop. Then, for $T_{\max }=t_{\max },=2 \mathrm{~s}$, the new discretised model results is:

$$
G_{x}(z)=\frac{1.288}{z}
$$

Therefore, as discussed in Section 2.1, it is necessary to evaluate the stability of the feedback system for the case of the maximum time between triggering instants, $t_{\max }$. The new loop gain of that feedback system results as shown in equation (14):

$$
G_{x} H(z)=0.386 \frac{z-0.802}{z(z-1)}
$$

And thus, its characteristic equation has two roots at $z=0.942$ and $z=-0.328$, giving a stable discrete system.

\subsection{Simulation}

In order to validate the design performed in Section 3.2, and compare the effect in the aperiodic solution of different triggering thresholds and reference signals, the following simulations are implemented.

Using the diagram in Figure 4, the mechatronic plant was evaluated taking into account its linear component (analysed in Section 3.1) and its nonlinear one, both identified with the reference period T (Losada et al., 2016). In such diagram, represented by Figure 5 , the plant input $\left(u_{k}\right)$ is the remote controller coded output ( 8 bits), and the plant output $\left(y_{k}\right)$ is the motion actuator speed (16 bits) in revolutions per minute (rpm).

Figure 5 Linear and nonlinear elements of the mechatronic plant model (see online version for colours)

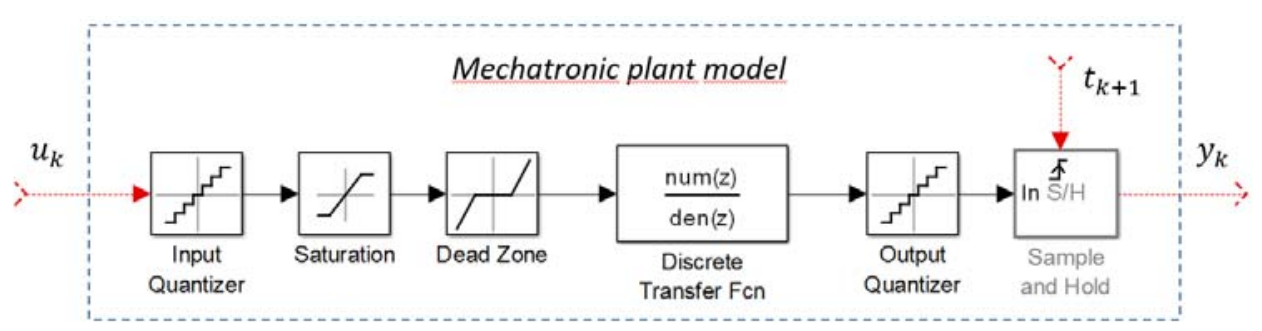

MATLAB simulation tools have been used to obtain the results commented in the following subsections, with step and ramp type reference signals (rpm). 


\subsubsection{Step type reference}

The test is performed for a time of $12 \mathrm{~s}$ and with a constant reference value $r_{T}=200 \mathrm{rpm}$ along $8 \mathrm{~s}$ in the middle of the simulation. Figure 6 shows the reference $r_{T}$ (black line), the model output $y_{T}$ (blue line), the output of the model preceded by the $\mathrm{S}$ and $\mathrm{H} \bar{y}_{T}$ (green line), the received response from the mechatronic plant (red line), and the triggering instants (blue circles), for a threshold $\delta=6$.

Figure 6 (a) Reference $r_{T}$ and output signals $\left(y_{T}, \bar{y}_{T}\right.$ and $\left.y_{k}\right)$ obtained from the simulation diagram in Figure 4 (b) The image is a zoom of the transient response (see online version for colours)

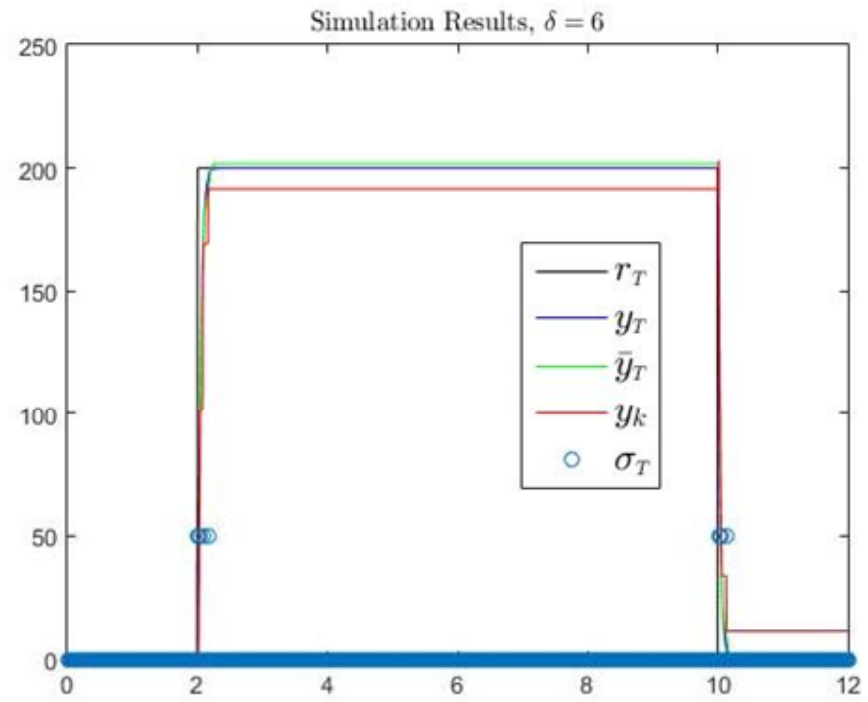

(a)

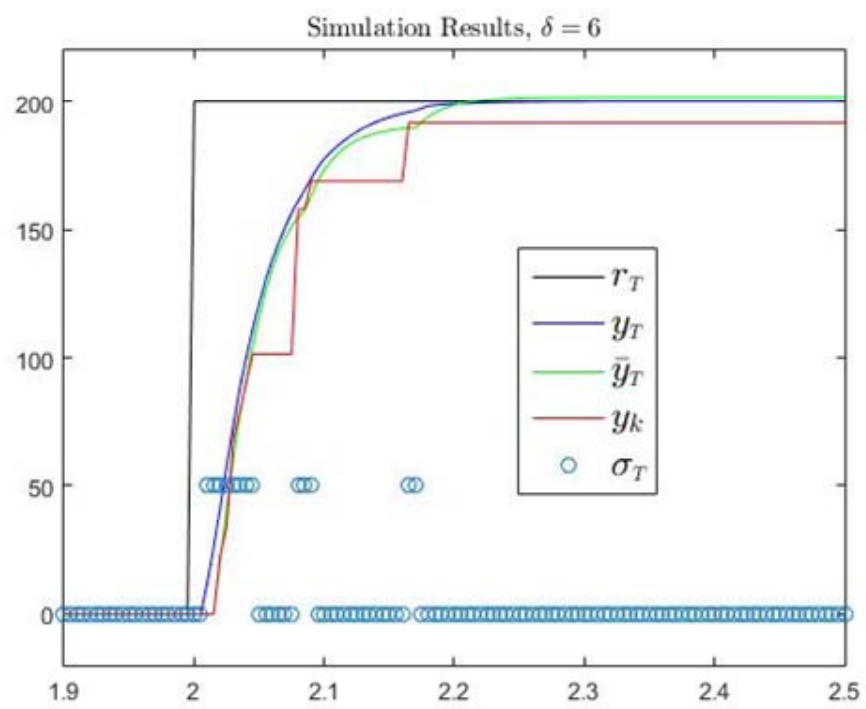

(b) 
Figure 7 (a) Error signal $\in_{T}$, triggering threshold $\delta$ and registered triggering events $\sigma_{T}$ occurred during the simulation (b) Actuation signals in the same simulation scheme (see online version for colours)

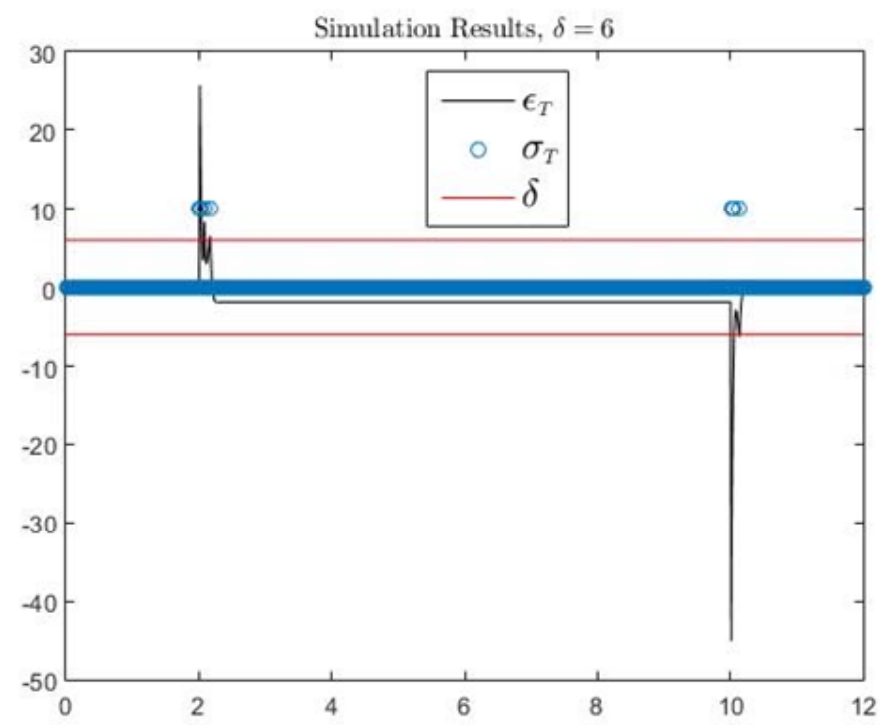

(a)

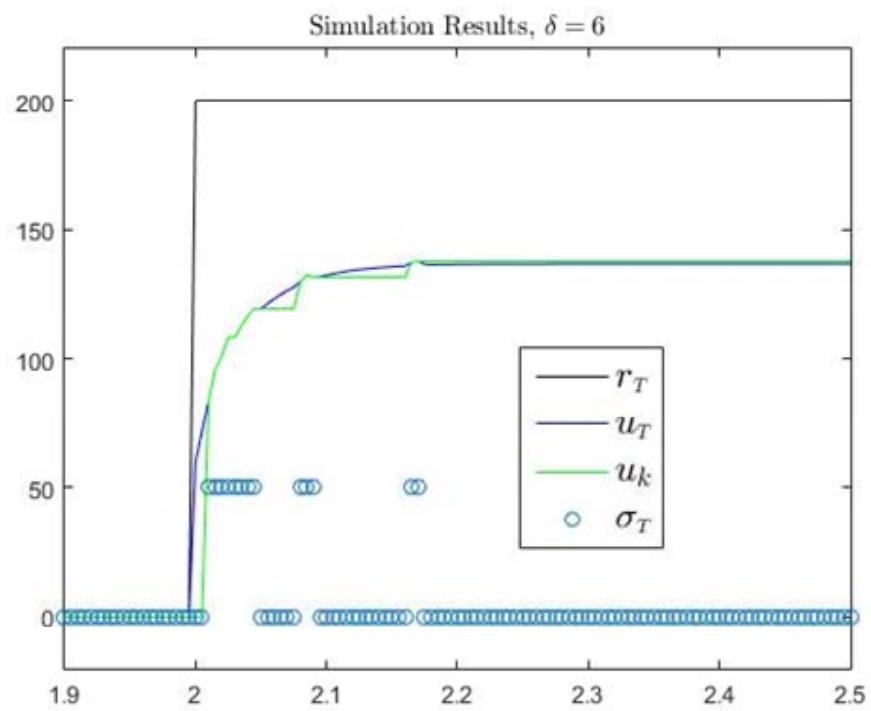

(b)

Note: See Figure 4 with periodic $u_{T}$, and aperiodic $u_{K}$ update.

As it can be notice in Figure 6, the triggering is activated only during the transient response derived from the reference changes. Only at event instants, controller and mechatronic plant communicate through the network channel. 
Figure 8 (a) Ramp type reference $r_{T}$ and outputs signals $\left(y_{T}, \bar{y}_{T}\right.$ and $y_{k}$ ), obtained from the simulation diagram in Figure 4 (b) Error signal $\in_{T}$, triggering threshold $\delta$ and registered triggering events $\sigma_{T}$ occurred during the simulation (see online version for colours)

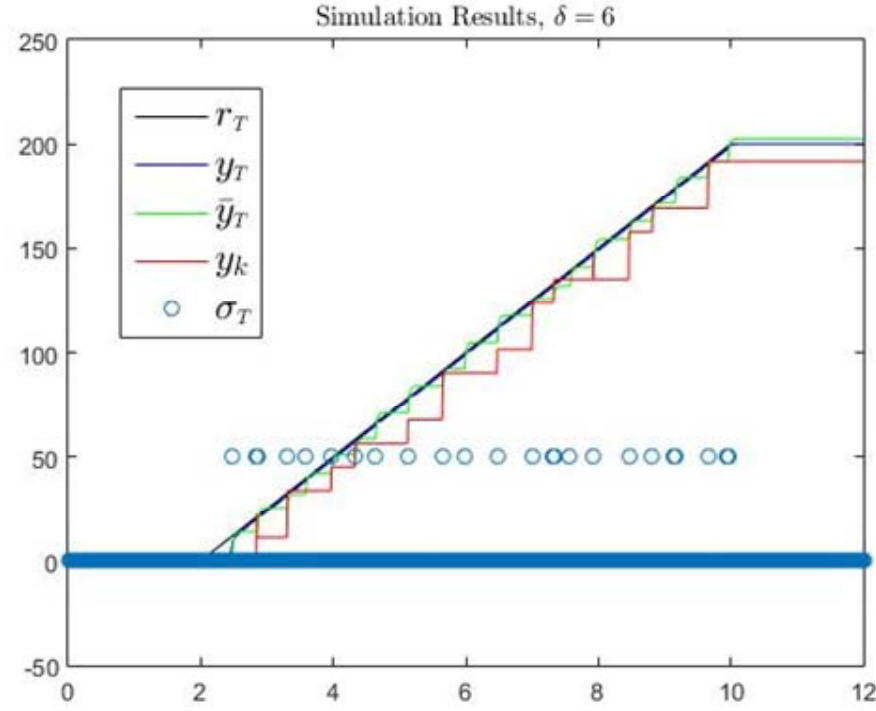

(a)

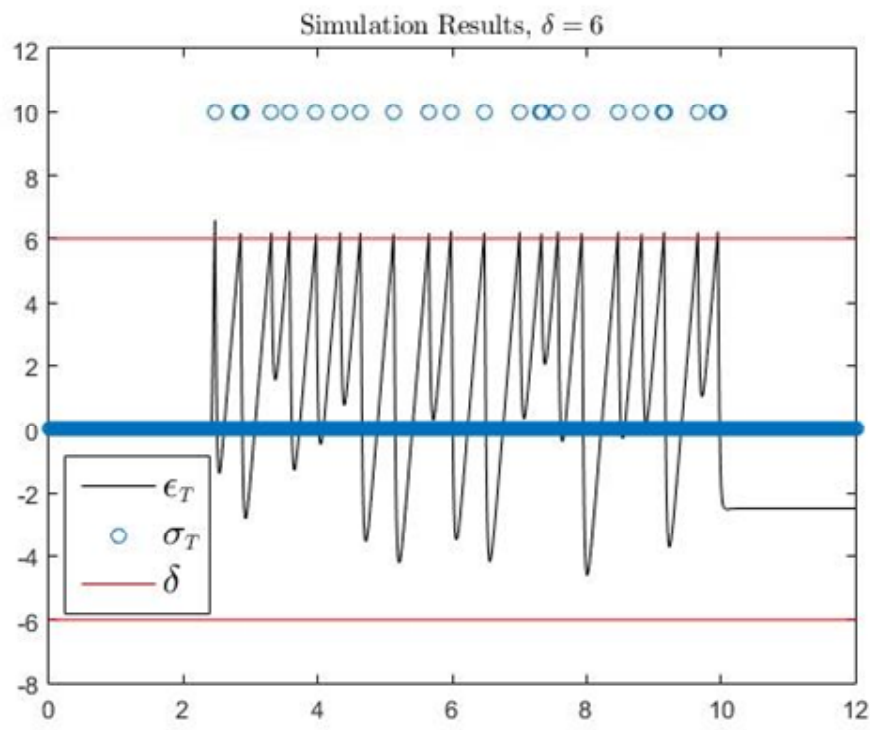

(b)

Figure 7 shows the error signal $\in_{T}$ (black line) corresponding to the triggering block input in Figure 4, as well as the triggering threshold $\delta$ (red line) and the generated triggering events $\sigma_{T}$. These events occur during the first $200 \mathrm{~ms}$ following each reference change. 
Therefore, the designer has to choose the parameter $\delta$ as a compromise between a minor reference tracking error $r_{T}-y_{k}$ (achieved reducing $\delta$ ), and a smaller number of triggering events (achieved increasing $\delta$ ).

\subsubsection{Ramp type reference}

Repeating the study with the same $\delta=6$ but with a ramp type reference characterised by a slope of $2.5 \mathrm{rpm} / \mathrm{s}$, we obtained the results shown in Figure 8 . As can be seen, with nonconstant references, the number of triggering events $\sigma_{T}$ increases, and the time between them tends to be periodic. As a first idea, we could say that the authors' proposal loses interest with varying time reference signals, but it is really true for unknown varying time ones due to the reference controller (Figure 3) can be redesigned for null steady state tracking error when known reference signals (step, ramp, parabolic, sinusoidal, etc.) are applied.

\section{Experimental results}

Once the theoretical proposal is validated through simulation, we prepared the real hardware setup in Figure 9 for experimental validation. The plant consists of an electric machine (specifically a DC-motor) with its electric drive, an electronic sensory interface and a communication module (Losada et al., 2016). The control device that implements the STC approach is a MiniDK2 development board with an LPC1768 processor, based on a Cortex-M3 core ([LPC, 2015, 2016). The MiniDK2 is connected with the interface board through a WLAN, as shown in Figure 2.

Figure 9 Elements in the real hardware setup for the STC experimental validation (see online version for colours)

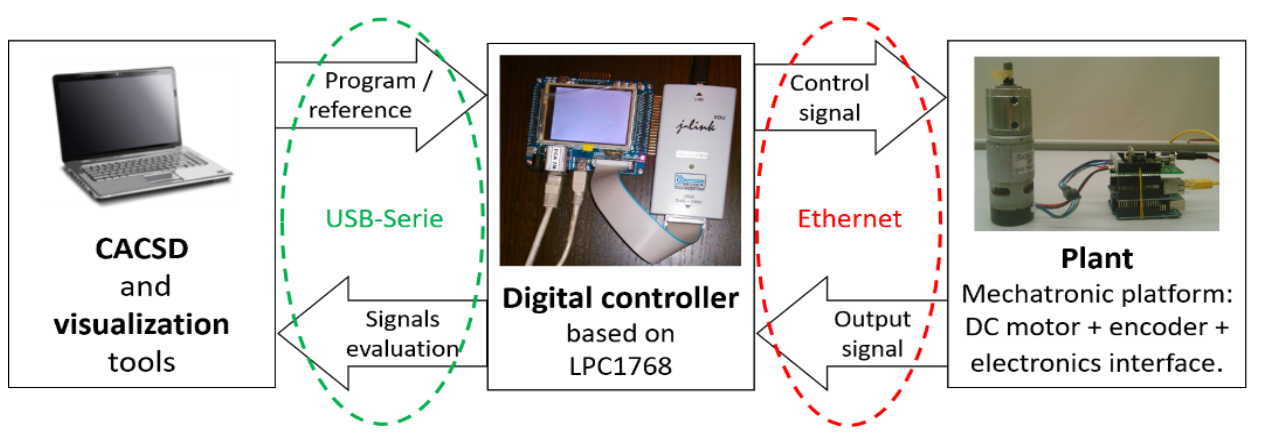

The MiniDK2 board is provided with a clock frequency of $100 \mathrm{MHz}$, an Ethernet interface, an USB HOST/device interface, an UART interface and a 16 bit parallel LCD interface. The USB interface is used as connection to a PC (Figure 9, left side), which facilitates the control solution development and the results evaluation.

Tests developed with this setup have two objectives. The first one is to obtain a complete model of the plant, for the reference period $\mathrm{T}$, being the plant tested in open loop. The second is to test the closed loop control system with different references and different triggering thresholds. 
The diagram in Figure 4 describes the STC control proposal and serves as a reference to program it in C. For simulation, compilation, debugging and downloading in the MiniDK2 board, Keil uVision four development environment, running in Windows $(\mathrm{C}$, is used.

As for the simulation (in Section 3.3), experimental results are presented for two reference types: step and ramp.

Figure 10 (a) Reference $r_{T}$ and output signals: $y_{T}, \bar{y}_{T}$ and $y_{k}$, registered in the STC control test applied to the plant in Figure 9 (b) The image is a zoom of the transient response (see online version for colours)

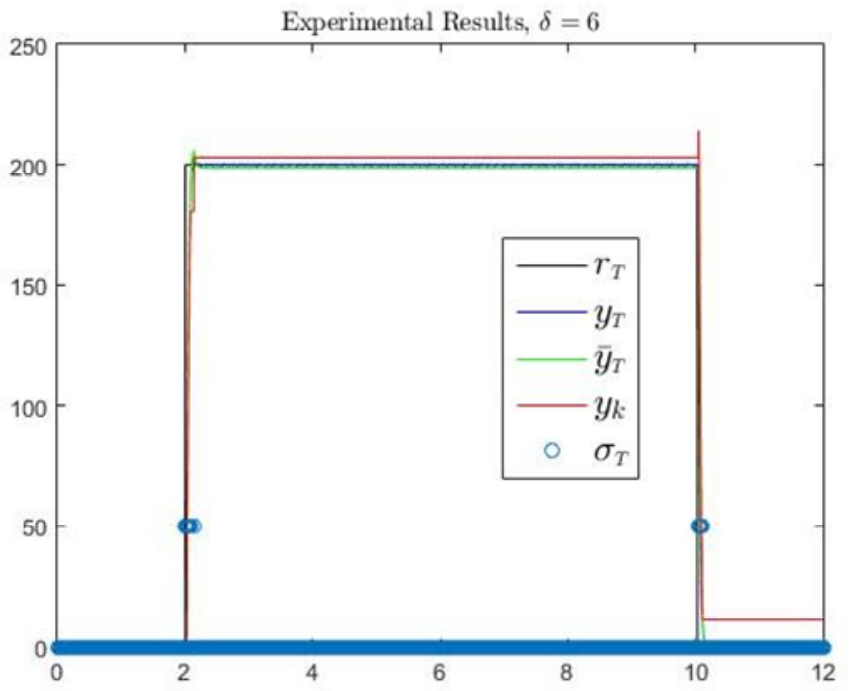

(a)

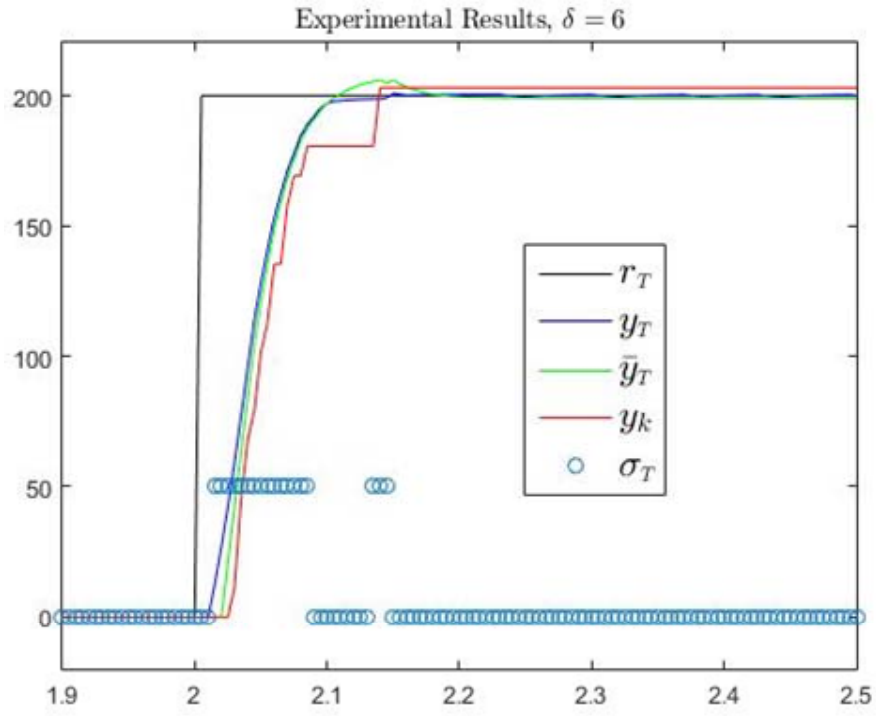

(b) 


\subsection{Step type reference}

The same step reference used in simulation (Subsection 3.3.1) has been chosen for real tests, and different triggering thresholds $(\delta: 2,4,6,12,20)$ have been tested.

Figure 11 (a) Error signal $\in_{T}$, triggering threshold $\delta$ and registered triggering events $\sigma_{T}$ occurred during the tests of the STC in the real platform (b) Actuation signals, in the same test, with periodic $u_{T}$ and aperiodic $u_{k}$ update (see online version for colours)

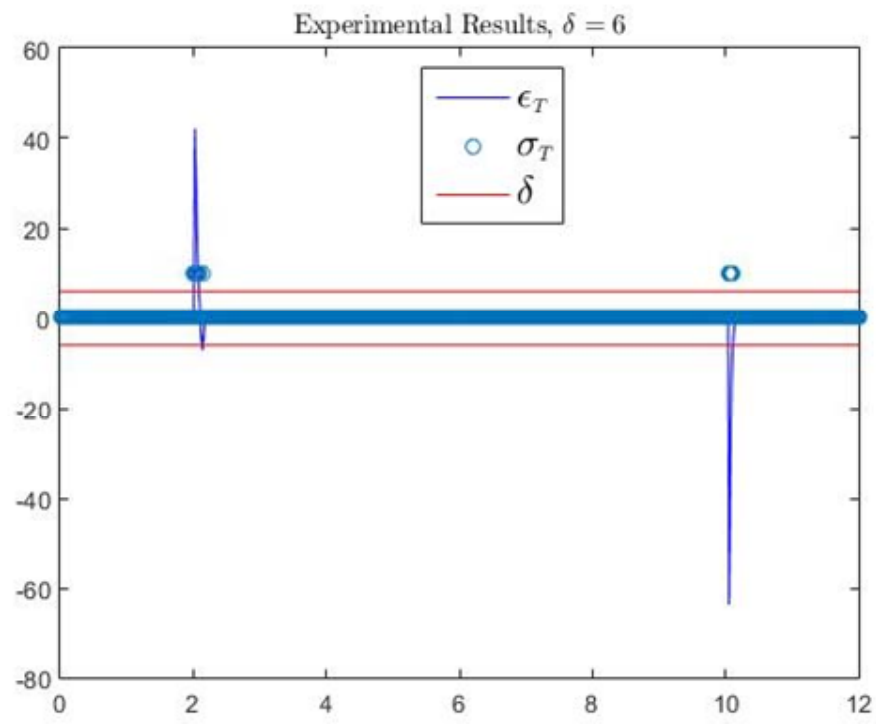

(a)

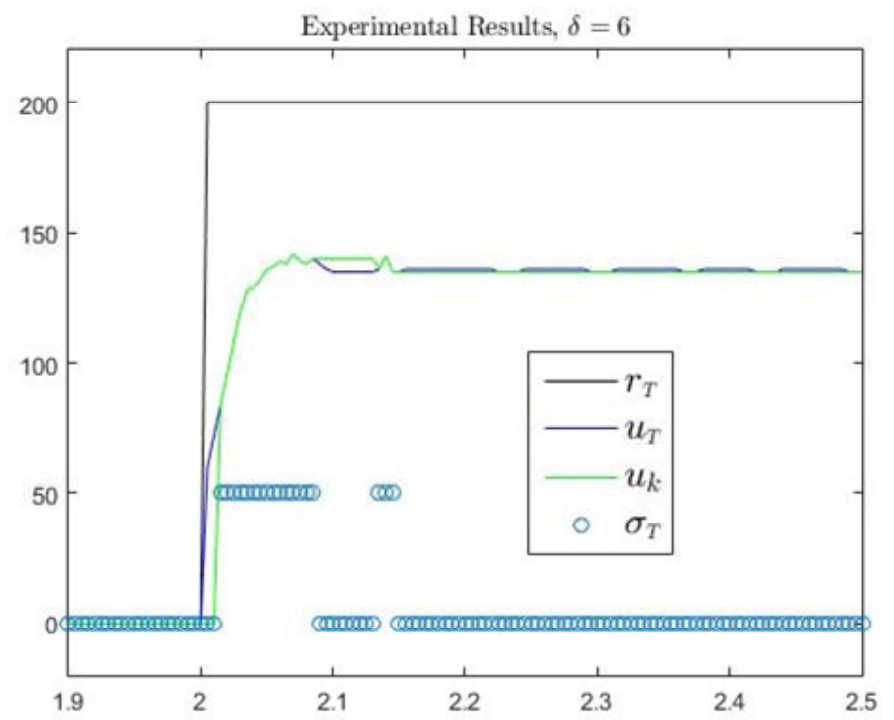

(b) 
Figure 12 (a) Ramp reference $r_{T}$ and output signals: $y_{T}, \bar{y}_{T}$ and $y_{k}$, obtained in the experimental test of the STC in the real platform (b) Error signal $\in_{T}$, triggering threshold $\delta$ and registered triggering events $\sigma_{T}$ occurred during the test (see online version for colours)

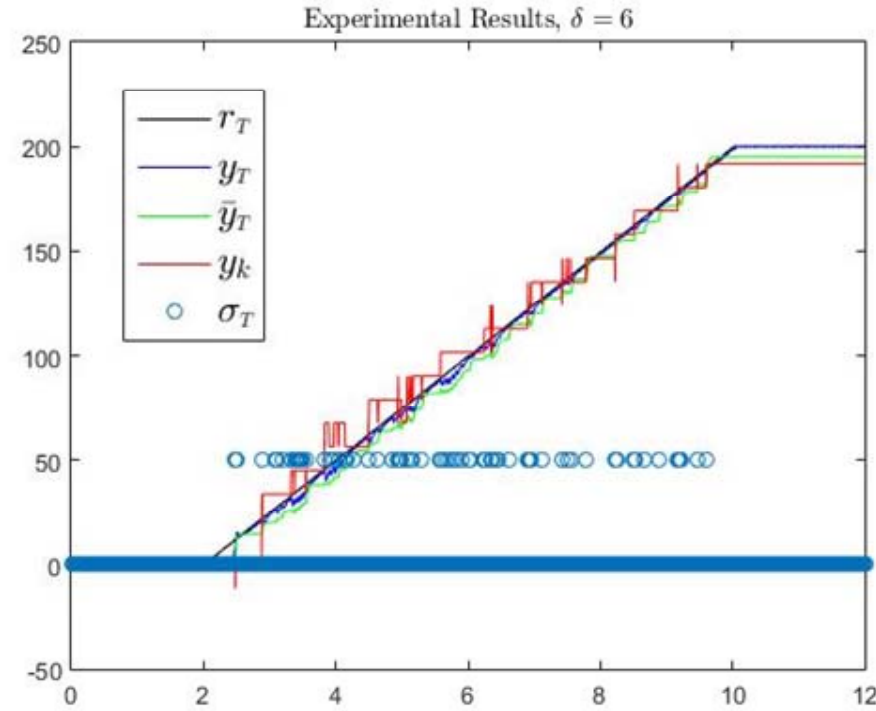

(a)

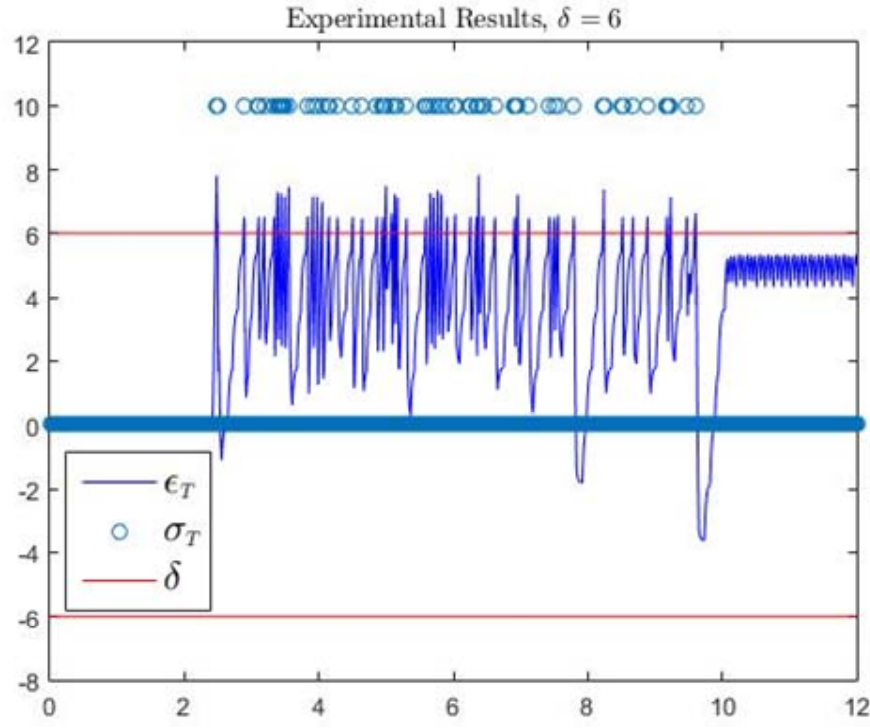

(b)

Figure 10 shows the reference $r_{T}$ (black line), the output $y_{T}$ of the model (blue line), the output of the model with $\mathrm{S}$ and $\mathrm{H} \bar{y}_{r}$ (cyan line), the response from the mechatronic plant (red line) and the triggering events (blue circles) for a triggering threshold $\delta=6$.

As it can be noticed in Figure 10, the number of triggering events recorded in response to the ascending step, between $t=2 \mathrm{~s}$ and $\mathrm{t}=10 \mathrm{~s}$, is 32 in the real test, 
meanwhile in the simulation is just 24 . Besides, this number increases to 1,600 in the periodic case (both when simulating and testing the algorithm with the real platform).

These results are very similar to those obtained in simulation. In the real implementation the number of triggering events generated in the transient increases slightly, allowing a closer approximation between the output $y_{k}$ and the reference $r_{T}$.

Figure 11 shows the signals recorded in the test, equivalent to the ones obtained in the simulation presented in Figure 7. Figure 11(a) shows the error signal $\in_{T}$ (black line), the triggering threshold $\sigma$ (red line), and the generated triggering events $\sigma_{T}$. Figure 11(b) shows the registers of the periodic $u_{T}$ (blue line) and aperiodic $u_{k}$ (green line) actuation signals are displayed. The slight increase in the number of triggering events, shown in Figure 11(a), justifies the smaller error $\epsilon_{T}$ in Figure 11(a), when compared with the one displayed with the simulation results [Figure 7(a)].

\subsection{Ramp type reference}

Taking advantage of the setup in Figure 9, the STC control is tested with the same $\delta=6$ and with a ramp type reference. The recorded results are shown in Figure 12. In this figure, it can be noticed the already commented effect: with a non-constant reference, the number of triggering events $\sigma_{T}$ increases, and the time between them tends to be periodic.

As it can be seen in Figure 12(a), the number of triggering events recorded between $\mathrm{t}=2 \mathrm{~s}$ and $\mathrm{t}=10 \mathrm{~s}$, is 159 in the real test, meanwhile in simulation is just 42, and with the periodic control rises to 1,600 . It has also to be noticed that in the periodic implementation of the control the number of accesses to the channel is independent of the reference type.

\section{Discussion, conclusion and future work}

Apart from the different graphical results presented in Section 4 for a specific triggering threshold $(\delta=6)$, tests for $\delta=2,4,6,12$ and 20 have also been performed. The effect of the triggering threshold is analysed below, focused on the experimental results obtained when applying a constant reference to the real mechatronic platform described in Figure 9. To quantify the tracking error in the mechatronic platform, the reference signal $r_{T}$ applied to it is compared with the output of this real plant, at sampling times.

The ISE index used to measure this tracking is shown in equation (15), resulting in the values shown in Table 1:

$$
I S E=\frac{1}{N} \sum_{K=1}^{N}\left(e_{k}\right)^{2}
$$

The results in Table 1 show that increasing the triggering threshold from 2 to 20 (units of the output variable of the mechatronic plant) reduces the number of triggering events to a value close to its half, while the ISE almost keeps unaltered (is multiplied by 1.05). That means, as expected, that an increase in the triggering threshold affects more the reduction of the number of communications between the plant and the remote controller, than the degradation of the control loop behaviour. 
Table 1 Effect of the triggering threshold on the number of channel accesses and ISE index in equation (14)

\begin{tabular}{lccc}
\hline \multirow{2}{*}{ Reference type } & \multicolumn{3}{c}{ Self-triggered control } \\
\cline { 2 - 4 } & Threshold value & $N^{\circ}$ of triggers & ISE \\
\hline \multirow{2}{*}{ Step } & 2 & 38 & 193.9944 \\
4 & 37 & 194.0867 \\
6 & 32 & 201.3477 \\
& 12 & 24 & 205.1908 \\
& 20 & 18 & 204.8796 \\
\hline
\end{tabular}

\subsection{Conclusions}

This paper presents a control demonstrator that facilitates the comparison within the classic remote periodic and aperiodic digital controller types over a real plant. It starts by presenting the periodic control of a specific mechatronic plant, discretised with a reference sampling period $\mathrm{T}$. The aperiodic solution is based on a connection mechanism between the controller and the real plant established by a triggering threshold to be determined by the designer. This threshold is placed upon the difference between the output of the reference model (when the periodic actuation signal is applied) and the signal from $\mathrm{S} / \mathrm{H}$ that represents the plant non-connected situation. When there is no connection with the plant, the necessary feedback for the control algorithm is obtained from the periodic reference model, but at the triggering instants that feedback is given by the output of the real plant.

Once the theoretical solution is described, the simulation of the control solution is presented, considering for the plant under study its identified model including linear and nonlinear elements (quantification, dead-zone and saturation). For experimental results the controller has been implemented in a MiniDK2 development board with an LPC1768 processor, based on Cortex-M3. As can be appreciated, they are slightly different from the ones predicted in simulation, as a consequence of a higher number of triggering events appearing in the real case, but lower, in any case, than the required ones in the periodic case.

The stability study has been simplified in this paper, taking into account that the reference control algorithm $H(z)$ is invariant, and that increasing the times between triggers is equivalent to discretising the plant with a sampling time higher than the reference one $\mathrm{T}$. In this way, for a sampling time between $\mathrm{T}$ and its maximum value established by the designer, the STC proposal stability can be analysed by applying the stability analysis usual techniques for linear systems.

This proposal is inspired in the self-triggered control, but periodically computing the internal variables of the control law. When the triggering mechanism decides, the actuation signal is sent to the mechatronic platform and the measurement signal is received in the remote control. The demonstrator helps to understand the strength of aperiodic solution when a communication network takes part of the closed loop: important reduction of the number of accesses to the communication channel with a slight degradation of the control quality. But it also shows the weakness of our proposal: the stability is only ensured for a range of sampling times. 
As future work, we will focus on designing an adaptation of the control structure for different reference signals and on anticipating the next triggering time $t_{k+1}$, this way the digital processor execution time is liberated to do other possible tasks such as statistical calculations, information visualisation, etc.

\section{Acknowledgements}

This work has been supported by the Spanish Ministry of Economy and Competitiveness through the ALCOR Project (DPI2013-47347-C2-1-R).

\section{References}

Anta, A. and Tabuada, P. (2010) 'To sample or not to sample: self-triggered control for nonlinear systems', IEEE Transactions on Automatic Control, September, Vol. 55, No. 9, pp.2030-2042.

Arzén, K.E. (1999) ‘A simple event-based PID controller', in Preprints IFAC World Conf., Vol. 18, pp.423-428.

Aström, K. and Bernhardsson, B. (1999) 'Comparison of periodic and event based sampling for first order stochastic systems', in Proc. IFAC World Conf., pp.301-306.

Dutton, K., Thompson, S. and Barraclough, B. (1997) The Art of Control Engineering, Pearson, England, ISBN: 970-0-201-17545-5.

Fadali, M.S. and Visioli, A. (2012) Digital Control Engineering: Analysis and Design, 2nd ed., September Elsevier, USA, ISBN: 978-0-12-394391-0.

Gupta, R.A. and Chow M.Y. (2010) 'Network control system: overview and research trends', IEEE Trans on Industrial Electronics, July, Vol. 57, No. 7, pp.2527-25-35.

Gupta, S. (1963) 'Increasing the sampling efficiency for a control system', IEEE Transactions on Automatic Control, Vol. 8, No. 3, pp.263-264.

Heemels, W.P.M.H., Johansson, K.H. and Tabuada, P. (2012) 'An introduction to event-triggered and self-triggered control', 51st IEEE Conference on Decision and Control, Maui, Hawaii, USA, 10-13 December 2012.

Heemels, W.P.M.H., Sandee, J.H. and Van Den Bosch, P.P.J. (2008) 'Analysis of event-driven controllers for linear systems', International Journal of Control, Vol. 81, No. 4, pp.571-590.

Heng, Z., Chen, P., Jin, Z. and Chu, Z. (2015) 'Event-triggered control in networked control systems: a survey', in Control and Decision Conference (CCDC), 2015 27th Chinese, May, pp.3092-3097.

Hespanha, J., Naghshtabrizi, P. and Xu, Y. (2007) 'A survey of recent results in networked control systems', Proceedings of the IEEE, January, Vol. 95, No. 1, pp.138-162.

Khalil, H.K. (2013) Nonlinear Systems, 3rd ed., Pearson, USA, ISBN-13: 978-1-292-03921-3.

Lehmann, D. and Lunze, J. (2009) 'Event-based control: a state-feedback approach', Proceedings of the European Control Conference 2009, Budapest, Hungary, 23-26 August 2009.

Losada, C., Espinosa, F., Santos, C., Gálvez, M., Bueno, E.J., Marrón, M. and Rodríguez, F.J. (2016) 'An experience of CACSD for networked control systems: from mechatronic platform identification to control implementation', IEEE Transactions on Education, 22 April, Vol. 59, No. 4, pp.1-8, DOI: 10.1109/TE.2016.2550586.

LPC176x Data Sheet (2015) [online] http://www.nxp.com/documents/data_sheet/LPC1769 68_67_66_65_64_63.pdf (accessed 11 July 2017).

LPC176x/5x User Manual (2016) December 2016 [online] http://www.nxp.com/documents/ user_manual/UM10360.pdf (accessed 11 July 2017). 
Lunze, J. (2014) Control Theory of Digitally Networked Dynamic Systems, Germany, ISBN 978-3-319-01131-8.

Martínez-Rey, M., Espinosa, F., Gardel, A., Santos, C. and Santiso. E. (2016a) 'Mobile robot guidance using adaptive event-based pose estimation and camera sensor', 2016 Second International Conference on Event-based Control, Communication, and Signal Processing (EBCCSP), DOI: 10.1109/EBCCSP.2016.7605089.

Martínez-Rey, M., Santiso. E., Espinosa, F., Nieto. R. and Gardel, A. (2016b) 'Smart laser scanner for event-based state estimation applied to indoor positioning', 2016 International Conference on Indoor Positioning and Indoor Navigation (IPIN), DOI: 10.1109/IPIN.2016.7743613.

Mazo Jr, M. and Tabuada, P. (2008) 'On event-triggered and self-triggered control over sensor/actuator networks', in 47th IEEE Conference on Decision and Control, 2008. CDC 2008, December, pp.435-440.

Mazo Jr, M., Anta, A. and Tabuada, P. (2009) 'On self-triggered control for linear systems: guarantees and complexity', in European Control Conference.

Miskowicz, M. (2015) Event-Based Control and Signal Processing, CRC Press, UK, ISBN: 9781482256550.

Ogata, K. (1995) Discrete-Time Control Systems, 2nd ed., Prentice Hall, USA, ISBN: 13 9780130342812.

Santos, C., Espinosa, F., Santiso, E. and Mazo Jr, M. (2015) 'Aperiodic linear networked control considering variable channel delays: application to robots coordination', Sensors 2015, Vol. 15, No. 6, pp.12454-12473, DOI: 10.3390/s150612454.

Santos, C., Mazo Jr, M. and Espinosa, F. (2014) 'Adaptive self-triggered control of a remotely operated P3-DX robot: simulation and experimentation', Robotics and Autonomous Systems, Vol. 62, No. 6, pp.847-854.

Sastry, S. (1999) 'Nonlinear Systems: Analysis, Stability and Control, Springer, USA, ISBN 0-38798513-1.

Slotine, J.E. and Li, W. (1991) Applied Nonlinear Control, Prentice-Hall, USA, ISBN 0-13040890-5 